RESEARCH COMMUNICATION

\title{
The GLUT5 hexose transporter is also localized to the basolateral membrane of the human jejunum
}

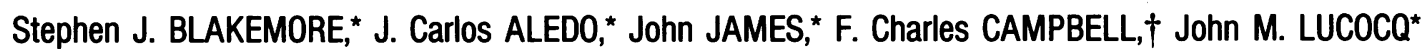 \\ and Harinder S. HUNDAL $¥$ \\ *Department of Anatomy and Physiology, University of Dundee, Dundee DD1 4HN, Scotland, U.K., and †Department of Surgery, \\ Ninewells Hospital and Medical School, Dundee DD1 9SY, Scotland, U.K.
}

The intestine is a major site of expression of the human GLUT5 hexose transporter, which is thought to be localized exclusively to the brush border membrane (BBM) where its major role is likely to be in the absorption of fructose. In this study we present novel biochemical and morphological evidence showing that the GLUT5 transporter is also expressed in the basolateral membrane (BLM) of the human intestine. BBM and BLM were isolated by fractionation of human jejunum. BBM were enriched with alkaline phosphatase activity by over 9-fold relative to a crude jejunal homogenate and contained immunoreactive sucrase-isomaltase and GLUT5 proteins. By contrast the BBM fraction was substantially depleted of immunoreactive al subunits of the Na,K-ATPase and GLUT2 glucose transporters which were abundantly present in the BLM fraction. This BLM fraction was enriched by over 11-fold in potassium-stimulated phosphatase activity relative to the crude homogenate; BLM also reacted to immunological probes for GLUT5 but showed no observable reactivity with antibodies directed against sucraseisomaltase. Quantitative immunoblotting revealed that the BBM and BLM contained near equal amounts of GLUT5 per mg of membrane protein. Immunogold localization of GLUT5 on ultrathin sections of human jejunum showed that GLUT5 was present in both apical BBM and BLM. This gold labelling was absent when antiserum was pre-incubated with the antigenic peptide corresponding to a specific $\mathrm{C}$-terminal sequence of human GLUT5. Quantitative analyses of the number of gold particles per unit length of BBM and BLM indicated that the mean density of gold labelling was marginally greater in the BBM ( 0.399 gold particles $/ \mu \mathrm{m})$ than in the BLM $(0.293$ gold particles/ $\mu \mathrm{m})$. The localization of GLUT5 in the BLM of the human jejunum may suggest that it specifically participates in the transfer of fructose across the basal membrane of the enteroctye.

\section{INTRODUCTION}

Fructose is a significant component of the normal human dietary sugar intake, but unlike glucose or galactose, which are absorbed across the luminal (apical) membrane of the intestine by the $\mathrm{Na}$ dependent glucose transporter (SGLT1), the uptake of fructose from the lumen is mediated by the GLUT5 protein, which belongs to the facilitative hexose transporter family (for review see [1]). The uptake of fructose across the apical brush border membrane (BBM) is 'supply-driven' with fructose being carried down its chemical gradient. The transfer of fructose across the basolateral membrane of the enterocyte has generally been assumed to be mediated by another member of this facilitative transporter family, the GLUT2 glucose transporter [2,3]. This assumption has largely been based on three observations. First, intestinal studies carried out in the rat have revealed that GLUT2 is the only hexose transporter that has so far been identified in the basolateral membrane of the jejunum. $[3,4]$. Secondly, liver, which represents a primary site of fructose metabolism, only expresses GLUT2 in its plasma membrane and is therefore likely to be responsible for liver uptake of fructose from the portal blood [4]. Thirdly, Xenopus oocytes expressing GLUT2, following micro-injection of human GLUT2 mRNA, display fructoseinhibitable glucose uptake [5]. However, oocyte expression studies have also revealed that GLUT2 displays markedly different transport kinetics for the two sugars, having a 6-fold higher affinity for glucose than for fructose [5]. Consequently, one would predict that the transfer of fructose across the human intestinal basolateral membrane may be limited by dietary glucose since this would be preferentially transported by GLUT2. However, since the flow of fructose is maintained down its concentration gradient the possibility exists that the basolateral membrane may express a second carrier, such as GLUT5, which may facilitate the transfer of fructose and possibly other hexoses across the basal membrane of the enterocyte. The present study has utilized a biochemical and morphological approach to test this proposition.

\section{EXPERIMENTAL PROCEDURES}

\section{Human jojunum}

Human jejunum (a 5-10 cm segment) was obtained from cadaver organ donors free of gastrointestinal disease. The total intestine was perfused in situ at the time of circulatory arrest, with cold $\left(4^{\circ} \mathrm{C}\right)$ intra-arterial Eurocollins solution [6]. Intestines were then removed and jejunal segments isolated and rapidly frozen in liquid $\mathrm{N}_{2}$ and subsequently stored at $-80^{\circ} \mathrm{C}$ until required for study. All jejunal tissue procured by this means had the approval of the local ethics committee.

\section{Isolation of BBM and basolateral membranes (BLM) from human jojunum}

Jejunal segments were thawed in wash solution containing $150 \mathrm{mM} \mathrm{NaCl}, 1 \mathrm{mM}$ Tris/Hepes (pH 7.5), $0.1 \mathrm{mM}$ phenyl-

Abbreviations used: BBM, brush border membrane; BLM, basolateral membrane; PMSF, phenylmethanesulphonyl fluoride; NP40, Nonidet P.40; KpNPPase, potassium-stimulated p-nitrophenyl phosphatase.

$\ddagger$ To whom correspondence should be addressed. 
methanesulphonyl fluoride (PMSF) and $1 \mathrm{mM}$ dithiothreitol. The segment was opened longitudinally, the luminal contents flushed off and the tissue maintained in the washing solution on an orbital shaker for 30 min with six changes of buffer solution. The protocol used to isolate BBM and BLM was based on two previously reported methods with some modification $[7,8]$. Briefly, mucosal scrapings were isolated and resuspended in homogenizing buffer $(0.25 \mathrm{M}$ sucrose, $0.1 \mathrm{mM}$ PMSF and $10 \mathrm{mM}$ triethanolamine $/ \mathrm{HCl}, \mathrm{pH}$ 7.6). The suspension was homogenized using a Polytron homogenizer (setting 7; $4 \times 15 \mathrm{~s}$ bursts) to yield a crude jejunal homogenate which was centrifuged at $2500 \mathrm{~g}$ for $10 \mathrm{~min}$. The resulting supernatant was removed and centrifuged at $19500 \mathrm{~g}$ for $20 \mathrm{~min}$. This spin results in a double pellet composed of a dark centre surrounded by a white 'fluffy' layer which represent crude jejunal membranes. The crude membranes were carefully removed and resuspended in buffer and homogenized (15 strokes at setting 12) in a Teflon/glass Potter homogenizer. This crude membrane suspension was diluted with Percoll (Sigma, Poole, Dorset, U.K.) to give a final Percoll concentration of $15 \%(\mathrm{v} / \mathrm{v})$. The mixture was re-homogenized and subsequently spun in a fixed-angle ultracentrifuge rotor at $48000 \mathrm{~g}$ for $35 \mathrm{~min}$. Two major bands were recovered from the Percoll gradient representing fractions enriched with BBM or BLM. These were isolated, washed by resuspension in buffer and subsequently re-pelleted by centrifugation at $190000 \mathrm{~g}$ for $60 \mathrm{~min}$. The pellet from the latter spin was then finally resuspended in $1 \mathrm{ml}$ of homogenizing buffer. Aliquots of homogenate $(0.5 \mathrm{ml})$, crude membranes, BBM and BLM were retained for enzymic and protein analysis. The protein content in each membrane fraction was measured using the Bradford method [9]. Alkaline phosphatase and potassium-stimulated $p$-nitrophenyl phosphatase (KpNPPase, an indicator of $\mathrm{Na}, \mathrm{K}-\mathrm{ATPase}$ activity) were assayed by standard enzymic methods [10].

\section{Human GLUT5 antiserum}

Polyclonal antibodies were prepared against human GLUT5 using a synthetic peptide corresponding to the C-terminal 14 amino acids of human GLUT5 (KEELKELPPVTSEQ) conjugated to BSA. Male Dutch rabbits were immunized with $1 \mathrm{mg}$ of conjugate/Freund's complete adjuvant (50:50) at five subcutaneous sites. Booster immunizations $(0.5 \mathrm{mg}$ of conjugate/ Freund's incomplete adjuvant) were administered over a 3 month period. The antibodies were affinity-purified on a peptideantigen-coupled Sepharose column (Pharmacia, Sweden) as per the manufacturer's instructions.

\section{Western-blot analyses}

To assess potential cross-reactivity of our purified GLUT5 antiserum against other glucose transporter isoforms, we immunoblotted human erythrocyte membranes, crude jejunal membranes, rat and human skeletal muscle plasma membranes and crude membranes from human liver and human brain microsomes. All membrane samples were subjected to SDS/ PAGE on either 7 or $9 \%$ polyacrylamide gels, essentially as described by Laemmli [11]. After electrophoretic transfer onto polyvinylidene difluoride membranes and blocking for $1 \mathrm{~h}[3 \%$ BSA/50 mM Tris-HCl, pH 7.2/150 mM NaCl/Tween 20/ Nonidet P.40 (NP40)]) samples were incubated overnight at 4-C in fresh affinity-purified GLUT5 antiserum $(1: 500)$ or that which had been pre-adsorbed with $0.5 \mathrm{mg} / \mathrm{ml}$ of the antigenic peptide. Membranes were washed three times (at $15 \mathrm{~min}$ intervals) in $50 \mathrm{mM}$ Tris- $\mathrm{HCl}$, pH 7.2/150 mM NaCl/Tween 20/NP40 and then incubated for $1 \mathrm{~h}$ with ${ }^{125} \mathrm{I}$-Protein $\mathrm{A}(0.1 \mu \mathrm{Ci} / \mathrm{ml})$ or with ${ }^{125} \mathrm{I}$-sheep anti-(mouse $\left.\mathrm{IgG}\right)(0.2 \mu \mathrm{Ci} / \mathrm{ml}$, for the monoclonal antibodies used, see below). All samples were then washed three times before air drying and autoradiography against Kodak XAR-5 film at $-80^{\circ} \mathrm{C}$.

Tissue homogenate, crude membranes, BBM and BLM (10 $\mu \mathrm{g}$ of protein from each) prepared from jejunal segments were immunoblotted for GLUT5 as described above. Jejunal membrane samples were also characterized immunologically using antibodies directed against sucrase-isomaltase (at a dilution of $1: 10$; provided by Dr. H.-P. Hauri, Basle, Switzerland) [12], to the $a 1$ subunit of the Na,K-ATPase (Mck-1 at a dilution of 1:100; provided by Dr. K. Sweadner, Harvard University, Boston, MA, U.S.A.) [13] and to the GLUT2 glucose transporter [anti-(human GLUT2) used at a dilution of 1:500 was provided by Dr. G. Gould, University of Glasgow, Scotland, U.K.]. Autoradiographs were quantified using a Molecular Dynamics laser densitometer with image quant 3 software.

\section{Electron microscopy}

Jejunal biopsies ( $\sim 50 \mathrm{mg}$ in total) were obtained from patients, who had previously undergone duodenal resection, during routine endoscopy investigations. Jejunal tissue was finely diced before fixation in $8 \%$ paraformaldehyde/0.2 M Hepes ( $\mathrm{pH} 7.2$ ). The tissue was cryoprotected by incubating in $2.1 \mathrm{M}$ sucrose/ PBS, pH 7.4, and then placed onto aluminium stubs for ultracryotome sectioning $(100 \mathrm{~nm})$. Sections were transferred onto Formivar/carbon-coated grids and incubated with affinitypurified GLUT5 antibody $(1: 10)$ for $20 \mathrm{~min}$ at room temperature. Grids $(8 \mathrm{~nm})$ were washed and then treated with Protein A-gold $(1: 30)$ [14] for $30 \mathrm{~min}$ at room temperature, washed and then stained with methylcellulose/uranyl acetate [15] before viewing using a JEOL 1200EX electron microscope. Immunogold labelling was quantified by applying standard stereological techniques to sets of systematic randomly sampled micrographs of the target membrane of interest as described previously [16].

\section{RESULTS AND DISCUSSION}

To assess the specificity of our affinity-purified GLUT5 antiserum we used it to probe membrane fractions prepared from rat and human tissues; no reaction occurred with membranes prepared from human erythrocytes, liver or rat skeletal muscle (Figure 1). These observations excluded the possibilities of cross-reaction with GLUT1, GLUT2 or GLUT4. However, discrete immuno-

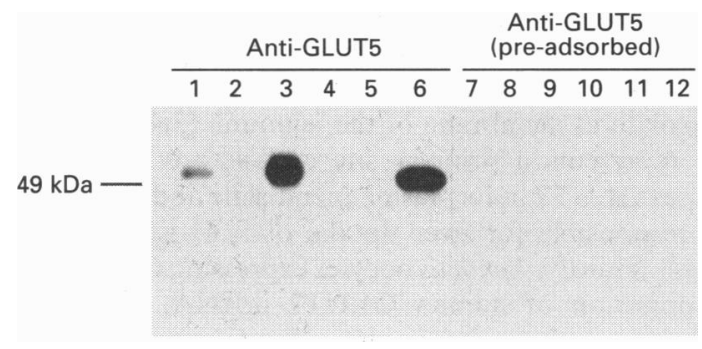

Figure 1 Western blot analyses showing the speclficlty of affinity-purfiled anti-GLUT5 antisera

Human brain microsomes (lanes 1 and 7), human erythrocyte membranes (lanes 2 and 8 ), crude jejunal membranes (lanes 3 and 9), crude human liver membranes (lanes 4 and 10) and plasma membranes prepared from rat skeletal muscle (lanes 5 and 11) and human skeleta muscle (lanes 6 and 12). Proteins $(10 \mu \mathrm{g})$ from each were subjected to SDS/PAGE and immunoblotting as described in the text. Samples in lanes 7-12 were immunoblotted with antiGLUT5 antibody that had been pre-adsorbed with the antigenic GLUT5 peptide. 


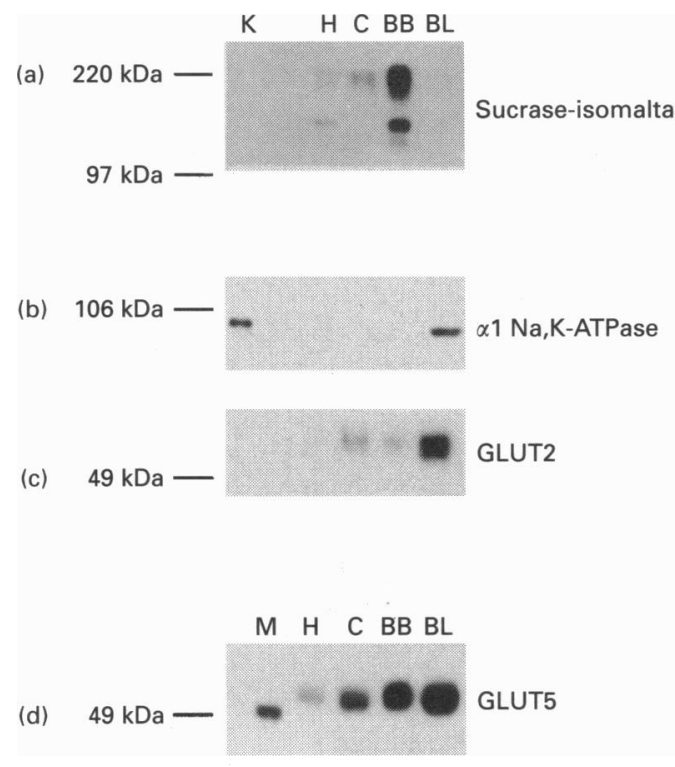

Figure 2 Representative Western blots showing the distribution of (a) sucrase-isomaltase, (b) a1 subunit of the Na,K-ATPase, (c) GLUT2 glucose transporter and (d) GLUT5 hexose transporter in jejunal homogenates (H), crude membranes (C), apical brush border membranes (BB) and basolateral membranes (BL)

Proteins $(10 \mu \mathrm{g})$ from each fraction were subjected to SDS/PAGE and immunoblotting as described in the text. Proteins $(10 \mu \mathrm{g})$ from rat kidney microsomes $(\mathrm{K})$ and plasma membranes prepared from human skeletal muscle $(M)$ were run in adjacent lanes as positive controls for the a1 subunit of the Na,K-ATPase and GLUT5 respectively $[13,18]$.

reactive bands of $\sim 55$ and $49 \mathrm{kDa}$ were observed in human jejunal crude membranes and in human sarcolemmal membranes respectively (Figure 1). These findings are in line with previous observations reporting the presence of GLUT5 in these tissues $[2,17,18]$. Human brain microsomes were also found to be weakly immunoreactive, consistent with the recent observations of Mantych et al. [19]. The immunoreactivity observed against human jejunal crude membranes, skeletal muscle and brain membranes was abolished when antiserum was pre-incubated with the antigenic peptide corresponding to the $14 \mathrm{C}$-terminal amino acids of human GLUT5 (Figure 1), providing further assurance of its specificity for the GLUT5 transporter.

To test the proposition that GLUT5 may be expressed in both the apical BBM and BLM of the human jejunum we initially characterized membrane fractions prepared from fractionating whole jejunal segments. Sucrase-isomaltase immunoreactivity was observed weakly in the homogenate and crude jejunal membranes but was strongest in the BBM; no detectable reactivity was observed in the BLM (Figure 2a). Another immunoreactive band was also observed at $\sim 200 \mathrm{kDa}$ in the BBM when these membranes were probed with the sucraseisomaltase antibody and most likely represents the position of the pro-enzyme [12]. Given that sucrase-isomaltase is a well established marker for the apical membrane, this observation signified that the BLM fraction was not contaminated to any significant extent with BBM. A monoclonal antibody directed against the al subunit of the $\mathrm{Na}, \mathrm{K}$-ATPase detected a single immunoreactive al protein band $(\sim 100 \mathrm{kDa})$ in rat kidney microsomes (which were used as a positive control). In our jejunal membrane samples the strongest al reactivity was observed in the BLM fraction but was undetectable in the BBM,



\section{Figure 3 Densitometric quantification of GLUT5 in apical BBM and in the BLM}

The relative peak areas per $\mu \mathrm{g}$ of protein obtained from three separate jejunal preparations were normalized to the GLUT5 signal obtained from plasma membranes prepared from human skeletal muscle (HM), run in adjacent lanes, assigned a relative value of 1.0. Since the protein yield of the BLM fraction was slightly higher than the BBM, the normalized BLM GLUT5 value was converted to total recovery by multiplying by the ratio of the total protein yield of BLM/BBM. The histogram bars represent the amount of GLUT5 present in the BBM and BLM recovered in each fraction, expressed in arbitrary units per gram of jejunum. The results are the means \pm SEM from three different preparations.

consistent with the notion that $\mathrm{Na}, \mathrm{K}-\mathrm{ATPase}$ is restricted to the basolateral membrane (Figure 2b). An analysis of the distribution of the human GLUT2 transporter revealed that it too was predominantly expressed in the BLM fraction (Figure 2c), consistent with the general view that this transporter facilitates glucose export across the basolateral membrane of the enterocyte $[3,4]$

Alkaline phosphatase activity was enriched by 9.3 -fold in BBM relative to the crude homogenate (from $32 \pm 5 \mu \mathrm{mol} / \mathrm{mg}$ of protein per $\mathrm{h}$ in homogenates to $299 \pm 81 \mu \mathrm{mol} / \mathrm{mg}$ of protein per $h$ in BBM, all values are mean \pm SEM of three separate jejunal preparations), whereas basolateral potassium stimulated KpNPPase activity was enriched more than 11.4-fold (from $1.4 \pm 0.4 \mu \mathrm{mol} / \mathrm{h}$ per $\mathrm{mg}$ of protein in homogenates to $16 \pm 3 \mathrm{mmol} / \mathrm{h}$ per $\mathrm{mg}$ of protein in BLM). In contrast the enrichment of $\mathrm{KpNPPase}$ activity in the $\mathrm{BBM}$ was $<1.0$ relative to that of the crude homogenate (BBM KpNPPase activity was $1.02 \pm 0.9 \mu \mathrm{mol} / \mathrm{h}$ per mg of protein). The KpNPPase enrichment ratio between the BLM and BBM was $\sim 16$, which is consistent with previous studies which have reported ratios of between 10 and 22.6 [7]. The above observations confirm that the two jejunal membrane fractions we isolated were significantly enriched with BBM and BLM with little cross-contamination.

When jejunal membrane samples were immunoblotted with our GLUT5 antiserum a single immunoreactive band ( $~ 55 \mathrm{kDa})$ was observed (Figure 2d) in all membrane fractions. This GLUT5 signal migrated at a slightly higher molecular mass than that obtained from plasma membranes prepared from human skeletal muscle and possibly reflects differences in GLUT5 glycosylation between the two tissues. Endoglycosidase F treatment of both jejunal and muscle membranes and subsequent Western blotting revealed that the native GLUT5 protein from both tissues migrated with the same molecular mass (results not shown). GLUT5 immunoreactivity in the jejunal samples was strongest in both the BBM and BLM fraction (Figure 2d). Interestingly, when the total protein yields of each fraction were taken into account (BBM $210 \pm 30 \mu \mathrm{g} / \mathrm{g}$ of jejunum; BLM $270 \pm 60 \mu \mathrm{g} / \mathrm{g}$ of jejunum) it was calculated that the BBM and BLM contained quantitatively similar amounts of GLUT5 per mg of membrane protein (Figure 3). This finding dispels the current notion that 



\section{Figure 4 Immunogold labelling of GLUT5 in ultrathin cryosections of human jejunum}

(a) Shows substantial gold labelling localized to membranes of microvilli (magnification $=\times 26000$ ); ( $b$ and $\mathbf{c}$ ) arrowheads show immunogold GLUT5 labelling localized to the basolateral membrane (magnification in $\mathbf{b}=\times 26000$, magnification in $\mathbf{c}=\times 39000$ ); ( $\mathbf{d}$ and $\boldsymbol{\theta}$ ) show the lack of any immunogold labelling of GLUT5 on apical and basolateral membranes (BL) respectively (magnification in $\mathbf{d}=\times 26000$, magnification in $=439000$ ). The position of the nuclear membrane $(\mathrm{N})$ and tight junctions $(\mathrm{T})$ are indicated (the scale bar represents $200 \mathrm{~nm}$ ).

the distribution of GLUT5 is polarized in the enterocyte and indicates that the protein appears to be targetted to both the BBM and BLM.

To verify that the GLUT5 signal observed in the BLM fraction by Western blotting was specifically due to its localization in basal membranes we performed immunogold labelling on ultrathin cryosections of human jejunum and examined them using electron microscopy. Immunogold GLUT5 labelling of both apical microvilli and basolateral membranes was detected (Figures 4a, 4b and 4c). We did not observe any significant labelling of other intracellular structures, such as the nuclear membrane or the outer membranes of mitochondria, ruling out non-specific reactivity of our GLUT5 antibody. Furthermore no immunogold labelling of BBM and BLM was observed when antiserum that had been pre-adsorbed with the antigenic peptide was used (Figures $4 \mathrm{~d}$ and $4 \mathrm{e}$ ) or when tissue grids were incubated with Protein A-gold alone with no prior exposure to our primary GLUT5 antibody (results not shown).

Quantification of the number of gold particles associated with the BBM and BLM per unit membrane length revealed that the labelling density over BBM and BLM (Table 1) was similar and in each case was more than 10 -fold higher than that over outer mitochondrial membranes. Previous quantitative studies suggest that the BBM and BLM contribute a similar fraction of the total membrane surface area of the enterocyte [20] and therefore it is likely, based on our biochemical and morphological data, that GLUT5 is present in both membranes in similar amounts. Indeed, some evidence is available supporting this proposition. In the human colonic adenocarcinoma cell line (Caco-2) it has been shown that GLUT5 was detectable in significant amounts in both the BBM and BLM [21]. However, given that these cells over-express both GLUT1 and GLUT3 [21], which are not 
Table 1 Quantification of GLUT5 immunogold labelling in human jojunum

Results are from a single piece of tissue. $n=$ Number of micrographs. Membrane lengths were calculated by applying standard stereological methods [16] to a set of micrographs that were positioned systematically over the tissue profile with a random start.

\begin{tabular}{llll}
\hline $\begin{array}{l}\text { Membrane } \\
\text { structure }\end{array}$ & $\begin{array}{l}\text { Signal density } \\
\text { (gold } / \mu \mathrm{m} \text { of membrane) }\end{array}$ & $n$ & $\begin{array}{l}\text { Coefficient of error } \\
\text { of a ratio estimate }(\%)\end{array}$ \\
\hline BBM & 0.399 & 21 & 13.6 \\
BLM & 0.293 & 19 & 16.1 \\
$\begin{array}{l}\text { Mitochondrion } \\
\text { (outer membrane) }\end{array}$ & 0.035 & 20 & 43.9 \\
\hline
\end{tabular}

present in the adult human intestine, and expression of GLUT5 appears to be related to cellular differentiation as well as the particular clone of Caco-2 cells being used [22], whether the observed distribution of GLUT5 in these tumour cells is fully representative of that in the normal human enterocyte remains unknown.

To our knowledge the present work represents the first biochemical and morphological demonstration that the GLUT5 transporter is also localized to the basolateral membrane of the human jejunal absorptive cells. Expression studies in oocytes indicate that human GLUT5 is a high-affinity fructose transporter with little capability of mediating glucose transport [2], and since human GLUT2 has a low $K_{m}$ for fructose compared with that for glucose [5] our results would lead us to suggest that in the human jejunum fructose transfer across the BLM is predominantly mediated by GLUT5, whereas the majority of the glucose flux occurs via GLUT2. However, a survey of the literature reveals that the question of whether GLUT5 can mediate the transfer of glucose has remained a controversial issue largely due to differences which have been observed between oocyte-based studies (utilizing GLUT5 clones from different species) $[2,23,24]$ and work carried out using intestinal membranes prepared from rodents [3,23]. For example, expression of rabbit GLUT5 in oocytes results in the appearance of D-glucoseinhibitable fructose uptake [23], a result that is distinct from that obtained using the human GLUT5 clone [2]. Moreover, this finding is inconsistent with the observation that in BBM vesicles prepared from rabbit jejunum, the uptake of fructose is not affected by D-glucose [23]. The reason for this apparent discrepancy is not clear but it has been suggested that the oocyte data may differ owing to the absence of a regulatory protein which may normally associate with GLUT5 and which substantially reduces its ability to transport glucose [23]. Studies in the rat are more suggestive that a difference in GLUT5 substrate specificity may exist between different species. For example, injection of rat GLUT5 mRNA in oocytes results in the expression of D-glucose-inhibitable fructose uptake [24]. Interestingly, rat GLUT5 expression also results in increased glucose uptake which can be inhibited by fructose, indicating that both sugars appear to compete for a common transporter [24]. The proposition that rat GLUT5 may possess a broader substrate specificity than human GLUT5 is further supported by circumstantial evidence which points to the existence of a second hexose carrier in the BLM of the rat intestine which may be involved in glucose/fructose transport. Rats made hyperglycaemic as a result of a $12 \mathrm{~h}$ glucose infusion display a marked adaptive increase in the $V_{\max }$ of intestinal BLM glucose transport [25]. The observed increase in glucose flux across the BLM is associated with a significant rise in cytochalasin B binding of the
BLM, indicating that there is a net increase in the total number of hexose transporters in the BLM [25]. However, Western-blot analyses reveals that the increase in cytochalasin B binding is unlikely to be attributable to GLUT2 since its expression in the BLM remains unaltered, thus implicating the involvement of a second carrier [25]. Cheeseman has also recently suggested that the activity of GLUT2 or a closely related second carrier is increased in BLM, on the basis that basolateral fructose transport could be rapidly up-regulated in response to an increased luminal glucose or fructose load [3]. However, in that study no carrier except GLUT2 was investigated and therefore the identity of any additional carrier(s) remained unknown.

The electron-microscopic localization of the GLUT5 transporter in basolateral membranes of the human jejunum supports our proposition that it is present on both luminal and bloodfacing membranes. The only previous morphological study localizing GLUT5 in the human intestine was at the opticalmicroscope level and reported that its expression was restricted to the BBM with little, if any, detectable GLUT5-labelling of the BLM [17]. The failure to detect GLUT5 using immunofluoresence is probably a consequence of the higher packing density of the microvillus membranes compared with basolateral ones. We have calculated from our electron micrographs that the packing of cylindrical microvillus membranes in three dimensions is approximately 5-fold higher than the packing of the closely opposed sheets of basolateral membranes. This means that a molecule such as GLUT5 which has similar density in the two membranes would produce a much stronger immunofluoresence signal from microvilli. The lower signal over the basolateral membranes may only become apparent in those regions where the basolateral membrane becomes folded, an idea that is supported by the previous observations of focal spots of fluorescence for GLUT5 in regions containing basolateral membranes [17].

In summary we have demonstrated both biochemically and morphologically that the GLUT5 hexose transporter is localized to both the apical and basolateral membranes of the human jejunum. Based on the available information in the literature with regard to its substrate specificity [2], it is very likely that the basolateral GLUT5 carrier specifically participates in the transfer of fructose across the basal membrane of the human enterocyte.

We are grateful to Dr. Peter Watt and Professor Michael Rennie for useful discussions and encouragement, and to Dr. K Sweadner, Dr. G. Gould and Dr. Hans-Peter Hauri for generously providing us with antibodies. S.J.B. is supported by a postgraduate studentship from the U.K. Sports Council and J.C.A. is an MRC postdoctoral research fellow. This work was supported by grants from SERC (J.M.L.), Medical Research Council (H.S.H.), The Royal Society (H.S.H.), The Wellcome Trust (H.S.H.) and The University of Dundee (H.S.H.). H.S.H. is the recipient of a Wellcome Trust University Award.

\section{REFERENCES}

1 Gould, G. W. and Holman, G. D. (1993) Biochem. J. 295, 329-341

2 Burant, C. F., Takeda, J., Brot-Laroche, E., Bell, G. I. and Davidson, N. O. (1992) J. Biol. Chem. 267, 14523-14526

3 Cheeseman, C. I. (1993) Gastroenterology 105, 1050-1056

4 Thorens, B., Cheng, Z.-Q., Brown, D. and Lodish, H. (1990) Am. J. Physiol. 259, C279-C285

5 Colville, C. A., Seatter, M. J., Jess, T. J., Gould, G. W. and Thomas, H. M. (1993) Biochem. J. 290, 701-706

6 Penny, J. I. and Campbell, F. C. (1994) Biochim. Biophys. Acta 1226, 232-236

7 Orsenigo, M. N., Tosco, M., Esposito, G. and Faelli, A. (1985) Anal. Biochem. 144, $577-583$

8 Wilde, S. W. and Kilberg, M. S. (1991) Biochem. J. 277, 687-692

9 Bradford, M. M. (1976) Anal. Biochem. 71, 248-254 
10 Bergmeyer, H. U. (1965) Methods of Enzymatic Analysis, Verlag Chemie/Academic Press, New York/London

11 Laemmli, U. K. (1970) Nature (London) 227, 680-685

12 Hauri, H. and Bucher, K. (1986) Anal. Biochem. 159, 386-389

13 Felsenfeld, D. P. and Sweadner, K. J. (1988) J. Biol. Chem. 263, 10932-10942

14 Lucocq, J. M. (1993) in Fine Structure Immunocytochemistry (Griffiths, G., ed.), pp. 279-302, Springer-Verlag, Berlin

15 Griffiths, G., McDowell, A., Back, R. and Dubochet, J. (1984) J. Ultrastruct. Res. 89, 65-84

16 Lucocq, J. M. (1994) J. Anat. 184, 1-13

17 Davidson, N. O., Hausman, A. M. L., Ifkovits, C. A. et al. (1992) Am. J. Physiol. 262, C795-C800
18 Hundal, H. S., Ahmed, A., Guma, A. et al. (1992) Biochem. J. 286, 348-353

19 Mantych, G. J., James, D. E. and Devasker, S. U. (1993) Endocrinology (Baltimore) 132, $35-40$

20 Buschmann, R. J. and Manke, D. J. (1981) J. Ultrastruct. Res. 76, 1-14

21 Harris, D. S., Slot, J. W., Geuze, H. J. and James, D. E. (1992) Proc Natl. Acad. Sci. U.S.A. 89, 7556-7560

22 Mesonero, J., Mahraoui, L., Matosin, M., Rodoloose, A., Rousset, M. and Brot-Laroche, E. (1994) Biochem. Soc. Trans. 22, 681-684

23 Miyamoto, K., Tatsumi, S., Morimoto, A. et al. (1995) Biochem. J. 303, 877-883

24 Rand, E. B., Depaoli, A. M., Davidson, N. O., Bell, G. I. and Burant, C. F. (1993) Am. J. Physiol. 264, G1169-G1176

25 Miyamoto, K., Takagi, T., Fujii, T. et al. (1992) FEBS Lett. 314, 466-470

Received 3 April 1995/21 April 1995; àccepted 9 May 1995 